\title{
Numerical Simulations of Nematic Liquid Crystals Flows between Eccentric Cylinders
}

\author{
Arash Nikzad, Dana Grecov \\ The University of British Columbia \\ 2329 West Mall, Vancouver, BC, Canada \\ arash.nikzad@mech.ubc.ca; dgrecov@mech.ubc.ca
}

\begin{abstract}
In this study, the flow characterization of Liquid Crystals (LCs) between eccentric rotating cylinders for different eccentricities is simulated using a finite element based commercial software COMSOL Multiphysics. To predict the interconnection of micro- and macro-structure of a Nematic Liquid Crystal (NLC), the Landau-de Gennes theory was implemented. Dimensionless pressure distribution and dimensionless wall shear stress on the inner cylinder is presented. Comparison of the mentioned dimensionless parameters for different eccentricities indicates that higher absolute values occur at higher eccentricity. Moreover, molecular representation of the NLC shows disclination lines and defects in the domain and also represent the preferred orientation of molecules near the solid surfaces.
\end{abstract}

Keywords: Numerical Simulation, Molecular Representation, Defects, Liquid Crystals, the Landau-de Gennes Theory.

\section{Introduction}

The efficiency of journal bearings is directly dependent on the lubricant performance. Lubricants affect the friction, wear, and load-bearing capacity. To assess the performance of a lubricant over a broad range of operating conditions, one can either conduct numerical simulation which is fast and economical or perform an experimental study which is a timeconsuming and expensive process.

Numerous experimental and simulation studies were carried out to consider both Newtonian and non-Newtonian fluids as a lubricant in both concentric and eccentric cylinders. Grecov and Clermont [1] examined the unsteady flow of incompressible non-Newtonian fluids in both concentric and eccentric rotating cylinders applying Stream-Tube Method (STM) and found significant viscoelastic effects in moderate and narrow gaps. Noroozi and Grecov [2] investigated the rheological behavior of a NLC between concentric cylinders, showing that Liquid Crystalline Materials (LCMs) used as lubricants reduce the resistance torque on the rotating cylinder compared with the Newtonian fluids.

LCMs are known to form an ordered molecular layer close to any solid boundary, which has been known to improve the tribological performance [3]. Considering the ability to be ordered near solid boundaries, as well as high load-carrying capacity, LCs could be used as lubricants in order to lower the friction coefficients, wear rates, and contact temperature of sliding surfaces which leads to an increase in the durability of the solid surfaces and saving energy [4].

To simulate the flow of LCs, several models have been introduced. One of the most complex continuum models is the Landau-de Gennes theory which has been presented in [5] and [6]. The Landau-de Gennes is a powerful model to simulate nematic phase of LCs in complex flows even with defects (which are non-singular solutions of the equations). This model works with order parameter tensor Q and the evolution of Q in time [7]. This evolution of microstructure depends on flow contribution as well as short- and long-range elasticity.

In this study, the main objective is to simulate the viscoelastic flow between eccentric cylinders for different eccentricities, which is a preliminary geometry for a journal bearing, using finite element based software COMSOL Multiphysics.

\section{Modelling}

To numerically simulate a rod-like LC between eccentric cylinders, mass, momentum, and evolution of order parameter tensor equations were solved. Applying the Landau-de Gennes model, the microstructure of LC, the formation, and the evolution of defects in the eccentric cylinder were studied. The evolution equation is defined by traceless and 
symmetric second order parameter tensor $(\mathrm{Q})$ describing the microstructure of LCMs. This tensor order parameter Q can be expressed as below [8] :

$$
\hat{Q}=F(\nabla v, Q)+H^{s r}\left(\bar{D}_{r}, Q\right)+H^{l r}\left(\bar{D}_{r}, \nabla Q\right)
$$

Where $\hat{Q}$ is the Jaumann derivatives of $Q$ and is defined by Eq.2. $F$ is the flow contribution, $H^{s r}$ and $H^{l r}$ are shortrange and long-range elasticity, $v$ is the velocity profile and $\overline{D_{r}}$ is the averaged microstructural rotational diffusivity.

$$
\hat{Q}=\frac{\partial Q}{\partial t}+(v \cdot \nabla) Q-W \cdot Q+Q \cdot W
$$

Where $W$ is the vorticity tensor, $W=\frac{1}{2}\left[\nabla v-(\nabla v)^{T}\right]$. Flow contribution at the microstructure level depends on $\mathrm{Q}$, shape parameter $\beta$, and the rate of the deformation tensor, $A=\frac{1}{2}\left[\nabla v+(\nabla v)^{T}\right]$. The shape parameter for high aspect ratio (length/diameter) rod-shape LCMs is 1.

$$
F=\frac{2}{3} \beta A+\beta\left[A \cdot Q+Q \cdot A-\frac{2}{3}(A: Q) I\right]-\frac{1}{2} \beta\{(A: Q) Q+A \cdot Q \cdot Q+Q \cdot A \cdot Q+Q \cdot Q \cdot A-[(Q \cdot Q): A] I\}
$$

$H^{s r}$ shows the inter-molecular forces and is defined by Eq.4 (Grecov \& Rey, 2003):

$$
H^{s r}=6 \bar{D}_{r}\left[\left(\frac{U}{3}-1\right) Q+U Q \cdot Q-U(Q: Q) \cdot\left(Q+\frac{1}{3} I\right)\right]
$$

Where $I$ is the unit tensor and $U$ is the nematic potential [9]. $H^{l r}$ represents the surface anchoring effects from the wall boundaries to the fluid [10]:

$$
H^{l r}=\frac{6 \bar{D}_{r} L_{1}}{c k_{B} T}\left\{\nabla^{2} Q+\frac{L^{*}}{2}\left[\nabla(\nabla \cdot Q)+[\nabla(\nabla \cdot Q)]^{T}-\frac{2}{3} \operatorname{tr}(\nabla(\nabla \cdot Q))\right]\right\}
$$

In Eq.5, $c$ represents the concentration of molecules in the liquid crystalline state; $K_{B}$ is the Boltzmann constant; $\mathrm{T}$ is the absolute temperature; $L^{*}=L_{2} / L_{1}$ and $L_{i}$ are the Landau coefficients.

The total stress tensor $\tau_{t}$ for a NLC has three main components which are the symmetric component $\tau_{s}$ - where $\tau_{s}=$ viscous $\left(\tau_{v}\right)+$ elastic $\left(\tau_{e}\right)$ - asymmetric component $\tau_{a}$, and Ericksen stress tensor $\tau_{E r}$ [6], [10].

The dimensionless numbers that are important in this numerical study are Ericksen number (Er), Deborah number (De), and Energy ratio (R) [3]:

$$
E r=\frac{c K_{B} T V h}{6 L_{1} D r} ; D e=\frac{V}{6 h D_{r}} ; R=\frac{c K_{B} T h^{2}}{L_{1}}
$$

$V$ is the velocity of the inner cylinder, $h$ is the flow characteristic length scale, $D_{r}$ is pre-averaged rotational diffusivity [11]. The dimensionless form of $\hat{Q}$ is shown below:

$$
\hat{Q}=F+\left(\frac{1}{D e}\right) H^{s r}+\left(\frac{1}{E r}\right) H^{l r}
$$

Furthermore, the dimensionless modified Navier-Stokes equation for a NLC can be written as [12]: 


$$
R e_{n} \cdot\left[\frac{\partial V^{*}}{\partial t^{*}}+\left(V^{*} \cdot \nabla^{*}\right) V^{*}\right]=-\nabla^{*} p^{*}+\nabla^{*} \cdot \tau_{t}^{*}
$$

Where $R e_{n}$ is a new definition of Reynolds number for LCs and equals to $R e_{n}=\rho V^{2} / c K_{B} T, V^{*}$ is dimensionless velocity, and $p^{*}$ is dimensionless pressure. The dimensionless total shear stress $\tau_{t}^{*}$ is $\left(\tau_{\ldots}^{*}\right.$ represents dimensionless total shear stress component):

$$
\tau_{t}^{*}=\frac{\tau_{t}}{c k_{B} T}=\tau_{a}^{*}+\tau_{e}^{*}+\left(\frac{E r}{R}\right) \cdot \tau_{v}^{*}+\left(\frac{1}{R}\right) \cdot \tau_{E r}^{*}
$$

Governing equations are continuity equation, Eq.7, and Eq.8, which are a set of six coupled non-linear PDEs. To simulate LCs in COMSOL Multiphysics, we used Laminar flow module (SPF) and PDE general module (G) in two dimensions. Furthermore, mesh independency and convergence of the final solution study were considered. The eccentricity ratio is $\left[\epsilon /\left(R_{2}-R_{1}\right)\right] \times 100$, where $\epsilon$ is the eccentricity and $R_{1}$ and $R_{2}$ are the inner and the outer cylinder radius respectively. The viscous stress tensor, $\tau_{v}^{*}$, in the Landau-de Gennes model includes three viscosity coefficients $\left(v_{i}^{*}\right)$ [10] which along with other required parameters in this study, are defined in the table 1 .

Table 1: Parameters used in this study.

\begin{tabular}{|c|c|c|c|c|}
\hline$E r=10^{8}$ & $D e=10$ & $R_{1}=0.7 \mathrm{~m}$ & $R_{2}=1 \mathrm{~m}$ & $R e_{n}=10^{-2}$ \\
\hline$L^{*}=0.45$ & $v_{1}^{*}=1$ & $v_{2}^{*}=-1$ & $v_{4}^{*}=6$ & Eccentricity ratio $=25 \%, 50 \%, 75 \%$ \\
\hline
\end{tabular}

\section{Results}

The effect of LCM microstructure on the flow properties on the inner cylinder for various eccentricity ratio at $R e_{n}=$ $10^{-2}$ and $t=1000 \mathrm{~s}$ (which is in the steady state condition), were presented in Fig.1 and Fig.2, which indicate the dimensionless pressure distribution $\left(P^{*}\right)$ and dimensionless wall shear stress $\left(\tau_{w}^{*}\right)$ on the inner cylinder. These figures show that increasing the percentage of eccentricity ratio leads to a higher absolute value of pressure distribution and wall shear stress on the inner cylinder. Besides, sharp variations in pressure and wall shear stress are occurring at the highest eccentricity ratio, while for the lowest eccentricity ratio the pressure distribution and wall shear stress are changing smoothly. Moreover, the highest eccentricity demonstrates not only sharper variations, but also a higher difference between extreme points.

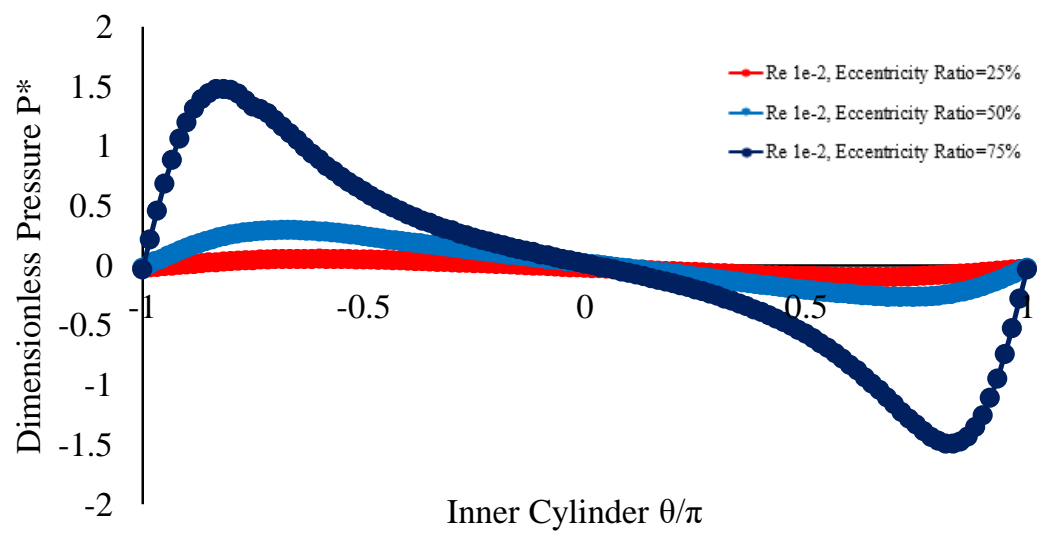

Fig. 1: Dimensionless pressure on the inner cylinder at $R e_{n}=10^{-2}$ and various eccentricities. 
In addition, the details of the microstructure of the LCM are presented using a molecular representation. The structure in the wider gap is magnified to offer a better visualization. Fig.3 shows the rod-like molecules for the eccentricity ratio of $75 \%$. As illustrated, defects and a disclination line in the texture of LC were captured by the simulation.

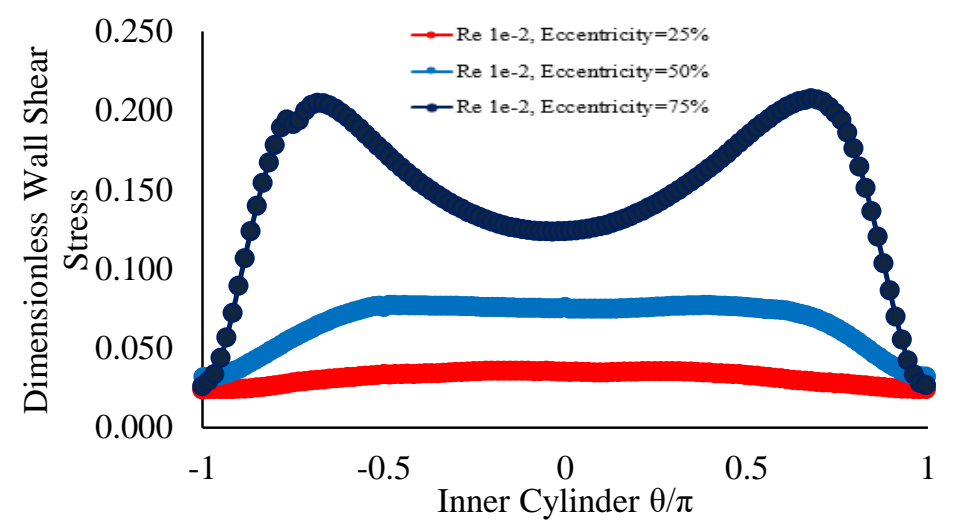

Fig. 2: Dimensionless Wall Shear Stress on the inner cylinder at $R e_{n}=10^{-2}$ and various eccentricities.

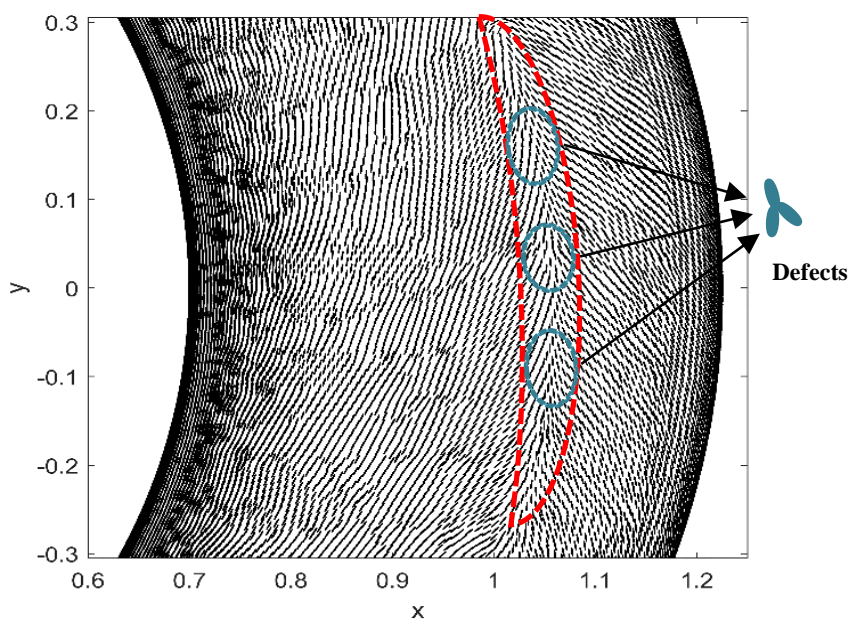

Fig. 3: Molecular representation of the LC in the wider gap of the domain at $\epsilon=75 \%$ and $R e_{n}=10^{-2}$.

\section{Conclusion}

Numerical simulation of NLC flow between eccentric rotating cylinders for three eccentricity ratios using the Landaude Gennes theory were presented. The results indicated that the magnitude of dimensionless pressure distribution and dimensionless wall shear stress on the inner cylinder increased with the eccentricity. The higher pressure difference in higher eccentricity translates into the higher load-carrying capacity for the LC. Molecular representation of LC was used for a better understanding of the molecular microstructure formation near the solid surfaces. The preferred orientation near the boundaries, defects, and disclination lines were observed in the simulation results.

\section{Acknowledgments}

Support from Compute Canada and from the Natural Sciences and Engineering Research Council of Canada, Discovery Grant, is gratefully acknowledged. 


\section{Reference}

[1] D. Grecov and J.-R. Clermont, "Numerical simulations of non-stationary flows of non-Newtonian fluids between concentric and eccentric cylinders by stream-tube method and domain decomposition," Rheol. Acta, vol. 47, no. 5-6, pp. 609-620, Jul. 2008.

[2] N. Noroozi and D. Grecov, "Flow modelling and rheological characterization of nematic liquid crystals between concentric cylinders," Liq. Cryst., vol. 40, no. 7, pp. 871-883, Jul. 2013.

[3] N. Noroozi and D. Grecov, "Numerical simulation of three-dimensional flow-induced microstructure in a simplified prosthetic hip joint with nematic liquid crystal lubricant," Rheol. Acta, vol. 53, no. 5-6, pp. 457-465, 2014.

[4] G. Biresaw, Tribology and the liquid-crystalline state, vol. 441. Washington, DC: American Chemical Society, 1990.

[5] Tsuji and Rey, "Effect of long range order on sheared liquid crystalline materials: flow regimes, transitions, and rheological phase diagrams," Phys. Rev. E. Stat. Phys. Plasmas. Fluids. Relat. Interdiscip. Topics, vol. 62, no. $6 \mathrm{Pt}$ A, pp. 8141-51, Dec. 2000.

[6] A. P. Singh and A. D. Rey, "Microstructure constitutive equation for discotic nematic liquid crystalline materials," Rheol. Acta, vol. 37, no. 1, pp. 30-45, Feb. 1998.

[7] D. Grecov and A. D. Rey, "Transient rheology of discotic mesophases," Rheol. Acta, vol. 42, no. 6, pp. 590-604, Nov. 2003.

[8] G. De Luca and A. D. Rey, "Chiral front propagation in liquid-crystalline materials: Formation of the planar monodomain twisted plywood architecture of biological fibrous composites," Phys. Rev. E, vol. 69, no. 1, p. 11706, Jan. 2004.

[9] A. Acevedo, P. M. Cotts, and Annette D. Shine, "Molecular weight dependence of the rotational diffusivity of rodlike polymers in concentrated nematic solutions," 2005.

[10] D. Grecov and A. D. Rey, "Transient rheology of discotic mesophases," Rheol. Acta, vol. 42, no. 6, pp. 590-604, Nov. 2003.

[11] J. L. Ericksen, "Conservation laws for liquid crystals conservation laws for liquid crystals," Cit. Trans. Soc. Rheol. J. Chem. Phys. I. Interfacial Free Energy J. Chem. Phys. J. Rheol., vol. 5, no. 34, pp. 1205-959, 1961.

[12] N. Noroozi, D. Grecov, and S. Shafiei-Sabet, "Estimation of viscosity coefficients and rheological functions of nanocrystalline cellulose aqueous suspensions," Liq. Cryst., vol. 41, no. 1, pp. 56-66, 2014. 